\title{
Annual health care resource utilization and cost among type 2 diabetes patients with newly recognized chronic kidney disease within a large U.S. administrative claims database
}

\author{
Kerstin Folkerts, MS; Natalia Petruski-Ivleva, PhD; Amanda Kelly, SM; Linda Fried, PhD; Michael Blankenburg, PhD; \\ Alain Gay, MD; and Csaba P Kovesdy, MD
}

\section{What is already known about this subject}

- Type 2 diabetes (TD2) is associated with a significant economic burden, exacerbated by costs incurred by complications and common comorbidities that include chronic kidney disease (CKD), anemia, and cardiovascular disease.

- Existing studies have used ICD-9-CM and ICD-10-CM diagnostic codes to identify CKD patients from administrative claims data, despite varying sensitivity and specificity of CKD algorithms.

- Studies using administrative claims data incorporating laboratory test results, which allow patient identification using the gold standard criteria from clinical practice, are scarce and the feasibility of such methods have not been documented.

\author{
What this study adds \\ - This study showed the ability to \\ successfully use administrative claims \\ data linked with laboratory data \\ (estimated glomerular filtration rate \\ or urine albumin-to-creatinine ratio) to \\ identify a CKD population, replicating \\ the gold standard criteria used in \\ clinical practice. \\ - Given the availability of laboratory test \\ results, results were more accurately \\ stratified by clinically relevant CKD \\ stage and showed that T2D patients \\ with advanced CKD had a 7-fold higher \\ rate of hospitalizations compared with \\ those with an early disease stage. \\ - This study also showed higher \\ health care resource utilization and \\ costs among patients with select \\ comorbidities, which could allow \\ stakeholders to identify patient \\ populations in need of targeted \\ interventions, including those for \\ comorbid conditions, to reduce health \\ care cost.
}

more scarce is evidence that takes into account disease severity and other comorbidities.

OBJECTIVES: To (a) describe T2D patients with CKD identified in U.S. administrative claims data using laboratory test results for kidney function that are considered the gold standard criteria for kidney disease diagnosis and (b) estimate the annual HCRU and costs

\author{
Author affiliations \\ Kerstin Folkerts, MS, Bayer AG, Wuppertal, \\ Germany; Linda Fried, PhD, University of \\ Pittsburgh, Pittsburgh, PA; and Csaba P \\ Kovesdy, MD, Division of Nephrology, \\ Department of Medicine, University of \\ Tennessee Health Science Center, Memphis. \\ Natalia Petruski-Ivleva, PhD, and Amanda \\ Kelly, SM, Aetion, Boston, MA. Michael \\ Blankenburg, PhD, and Alain Gay, MD, \\ Bayer AG, Berlin, Germany. \\ AUTHOR CORRESPONDENCE: \\ Csaba P Kovesdy, 901.448.5764, \\ ckovesdy@uthsc.edu \\ J Manag Care Spec Pharm. \\ 2020;26(12):1506-16 \\ Copyright $\odot 2020$, Academy of Managed \\ Care Pharmacy. All rights reserved.
}

among these patients, overall and by disease severity and comorbidity subgroup.

METHODS: Optum CDM data between the years 2008 and 2017 were used to identify T2D patients with newly recognized CKD, using laboratory test results for estimated glomerular filtration rate (eGFR) or urine albumin-to-creatinine ratio (UACR). The study estimated annualized total, inpatient, 
outpatient, and pharmacy costs and the number of outpatient, inpatient, and emergency room visits in the first year after CKD identification. Analyses were stratified by prevalent anemia, heart failure (HF), resistant hypertension, and by CKD stages.

RESULTS: T2D patients with newly recognized CKD $(n=106,369)$ had a high prevalence of cardiovascular comorbidities and incurred on average $\$ 24,029$ of total cost per person per year in the first year after CKD identification. Patients with HF and anemia incurred on average $\$ 41,951$ and $\$ 31,127$ of total annual cost, respectively. Patients identified at stage 5 CKD incurred on average $\$ 110,210$ of total annual cost and had roughly a 7-fold higher annual inpatient hospitalization rate compared with patients identified at stage 1 CKD.

CONCLUSIONS: Administrative claims data linked to laboratory results provide an opportunity to identify CKD patients using the gold standard criteria from clinical practice, minimizing potential misclassification of patients. Identified CKD patients, particularly those with $\mathrm{HF}$, anemia, and more advanced CKD stage, incur high HCRU and cost. Better monitoring, earlier CKD diagnosis, and interventions that are effective in halting or slowing the progression of CKD, as well as at managing comorbid conditions, could be effective means to reduce the economic burden of CKD in T2D.

Type 2 diabetes mellitus (T2D) is an increasingly prevalent disease with 23.1 million people diagnosed in 2017 in the United States alone. ${ }^{1}$ Many patients with diabetes display multimorbidity, which results in significant burden on patients' health and health systems. ${ }^{2}$ As a result of this high morbidity, T2D has been associated with a significant economic burden, with an estimated cost of \$327 billion in 2017 because of direct medical costs and reduced productivity. ${ }^{3}$

Chronic kidney disease (CKD) is one of the most common complications of T2D and occurs in $20 \%-40 \%$ of diabetic patients. ${ }^{4-8}$ Costs incurred by patients with kidney disease and T2D and/or heart failure (HF) accounted for more than $50 \%$ of all Medicare costs in 2015; overall health care expenditures increased as kidney disease severity increased. ${ }^{9}$ Moreover, common comorbidities observed in $\mathrm{CKD}$ and $\mathrm{T} 2 \mathrm{D}$, including $\mathrm{HF}$, anemia, and resistant hypertension $(\mathrm{RH})$, have each been shown to lead to worse health outcomes related to CKD..$^{10-14}$ Annual spending for those with CKD, T2D, and HF was more than twice that for patients with CKD alone. ${ }^{15}$

Contemporary studies describing T2D patients with CKD in real-world care settings are scarce, and even more limited are studies using real-world data that identify patients using the gold standard criteria of estimated glomerular filtration rate (eGFR) and urine albumin-to-creatinine ratio (UACR). While most existing studies use International
Classification of Diseases, Ninth/Tenth Revision, Clinical Modification (ICD-9/10-CM) diagnostic codes to identify CKD patients from claims data, ICD-9/10-CM codes show suboptimal performance in identifying CKD. ${ }^{16}$ Kern et al. (2006) estimated the sensitivity of algorithms using ICD-9-CM codes to be between $13.3 \%$ and $42.4 \%$ when validated against laboratory test results. ${ }^{16}$ Many patients were captured as "CKD, unspecified," which did not provide information on stage of the disease. Thus, the objective of this study was to estimate annual health care resource utilization (HCRU) and costs of T2D patients with newly recognized $C K D$, identifying patients from a U.S. claims database using laboratory test results. In addition, results were presented for subgroups of patients with anemia, $\mathrm{HF}$, and RH, as well as stratified according to the Kidney Disease: Improving Global Outcomes (KDIGO) CKD stage for CKD severity.

\section{Methods}

\section{DATA SOURCE}

This study was designed as a retrospective cohort study using the deidentified Optum Clinformatics Data Mart (CDM) database (OptumInsight, Eden Prairie, MN), with data collected from January 1, 2007, through December 31, 2017. This administrative claims database is composed of longitudinal data of patients enrolled in a commercial or Medicare Advantage health plan and includes information on demographics, medical encounters from inpatient and outpatient settings, pharmacy dispensing, and laboratory test results for a subset of patients. With approximately 63 million unique members, this database is representative of the commercially insured U.S. population with respect to age, gender, and region, allowing generalizability of findings. ${ }^{16}$

\section{STUDY POPULATION}

Patients were eligible to enter the cohort at any time between January 1, 2008, and December 31, 2017 (Figure 1). The cohort included T2D patients aged over 18 years who met the criteria for newly recognized $\mathrm{CKD}$, defined as at least 2 laboratory results for eGFR of $<60 \mathrm{~mL} / \mathrm{min} / 1.73 \mathrm{~m}^{2}$ or at least 2 results for UACR of values $\geq 30 \mathrm{mg} / \mathrm{g}$ between 90 to 365 days apart. ${ }^{17-20}$ Two results in the CKD range between 90 and 365 days apart were required to avoid capturing acute kidney failure patients. The second laboratory result confirming CKD was used as the index date and to assign the stage of CKD on index. 


\section{FIGURE 1 Study Design}

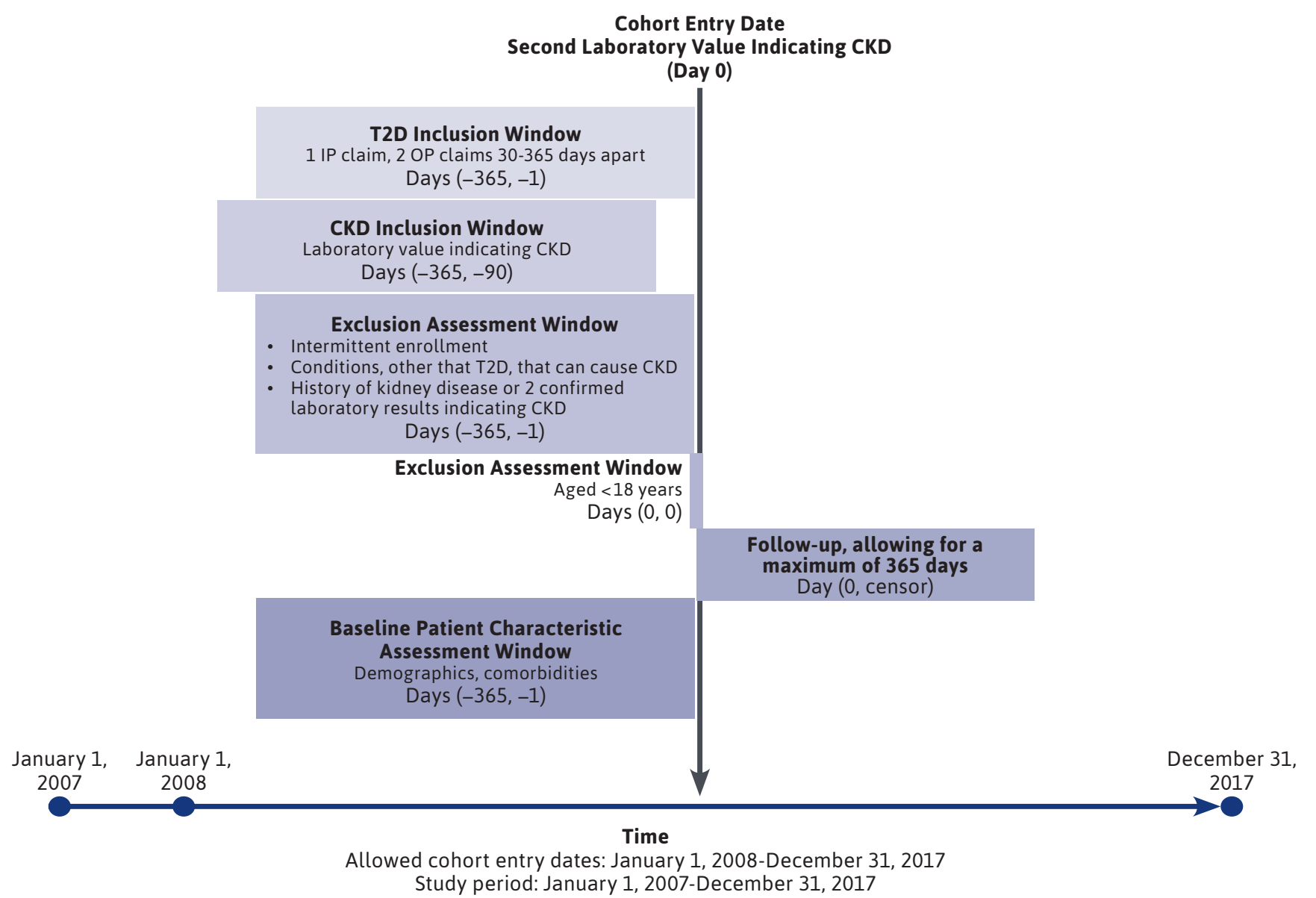

$C K D=$ chronic kidney disease; $I P=$ inpatient; $O P=$ outpatient; $T 2 D=$ type 2 diabetes

Values with incorrect units, those outside of plausible range, and observations that had multiple discordant results on the same day were not considered for cohort entry. These steps excluded $<1 \%$ of observations. Patients were excluded if they showed evidence of kidney disease at baseline as indicated by at least 2 laboratory results in the CKD range; at least 1 claim with ICD-9/10-CM diagnosis codes indicating CKD; evidence of dialysis, end-stage renal disease (ESRD), or kidney transplant; or those with claims with diagnostic codes that could indicate kidney disease from causes other than T2D. ${ }^{21}$

All patients were required to have a T2D diagnosis at baseline, defined as at least 1 inpatient or 2 outpatient ICD-9/10-CM diagnosis codes for T2D at least 30-365 days apart. Patient were required to have a minimum of 365 days of baseline enrollment before cohort entry date. A full list of definitions is located in Supplementary Materials 1: Definitions (available in online article).

\section{PATIENT CHARACTERISTICS}

Demographic information, prevalent comorbidities, and medication use were assessed using medical and pharmacy claims during the 365-day baseline period before cohort entry. Comorbidities were considered present if there was at least 1 medical claim with an ICD-9/10-CM diagnosis code for the condition during baseline. Patients were considered users of a medication if they had at least 1 claim for the medication in baseline. 


\section{FIGURE 2 Flowchart of Patient Selection for Patients with T2D and Incident CKD}

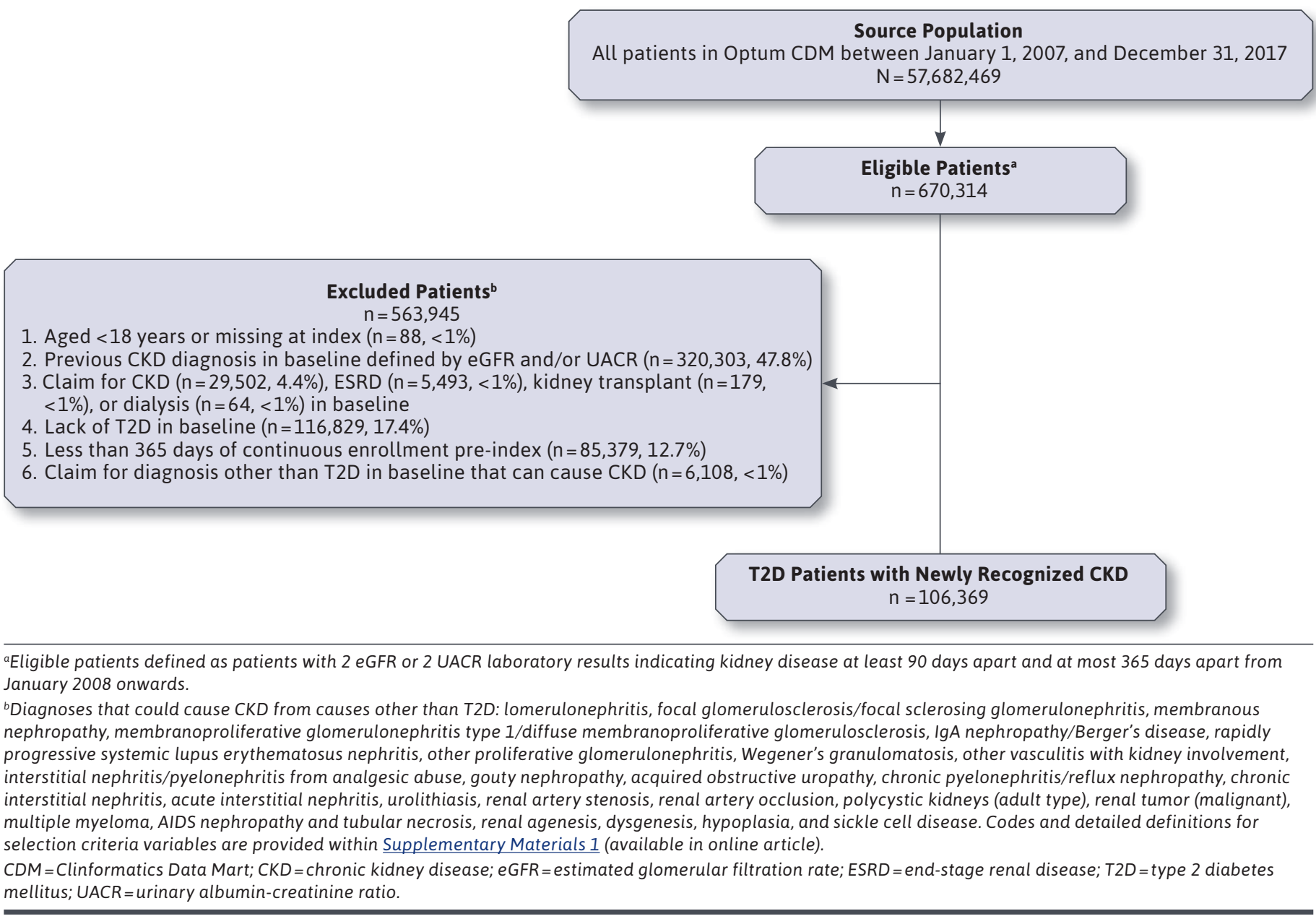

\section{OUTCOMES}

HCRU was measured in terms of the number of inpatient, urgent care, outpatient, and emergency room (ER) visits during follow-up. The mean duration of all inpatient stays was also assessed. Standard costs were measured as total costs during follow-up and stratified according to inpatient, urgent care, outpatient, ER, and pharmacy costs. Costs before the year 2014 were normalized to year 2014 to adjust for inflation; costs from 2014 and onward were left unadjusted. All outcomes were assessed in follow-up beginning with the day of cohort entry and ending at the end of data (December 31, 2017), disenrollment, death, or a maximum of 365 days of follow-up.

To account for differences in pricing across health plans and provider contracts, Optum applies standard pricing algorithms to the claims data. These algorithms are designed to create standard prices that allow for comparisons across patients, data sources, and geographic areas in a consistent manner, as if the data were derived from a single source using a single approach for classifying and pricing services.

\section{STATISTICAL ANALYSIS}

Baseline characteristics were assessed in the 365 days before the index date. Means and SDs were reported for continuous covariates, and frequencies and percentages were reported for binary or categorical covariates.

HCRU and cost outcomes were annualized and reported as per person per year (PPPY) for the first year after index following the method used by the United States Renal Data System. ${ }^{22}$ The analysis was not restricted to patients with 
365 days of follow-up; however, follow-up was capped at 365 days. This approach thus included patients who died or disenrolled from their health plans before 365 days of follow-up. To annualize HCRU counts, the sum of HCRU events for all patients in the cohort was divided by the total number of days of person-time that contributed to followup, and then multiplied by 365 days to reach an estimate of the mean count PPPY. To annualize costs, the sum of all costs for all patients in the cohort was divided by the total number of days of person-time that contributed in followup and then multiplied by 365 days to create an estimate of the mean cost PPPY.

All analyses were stratified by prevalent HF, RH, and anemia evaluated in baseline. RH was defined as 4 or more unique antihypertensive medication prescription claims within a 30-day period. Each of these conditions has been shown to lead to worse health outcomes related to CKD and increased health resource utilization and costs. ${ }^{11}$

Analyses were also stratified by KDIGO CKD stages 1-5 (stage 1 [G1], >90 ml/min/1.73m²; stage 2 [G2], 60-89 ml/ $\mathrm{min} / 1.73 \mathrm{~m}^{2}$; stage $3 \mathrm{a}$ [G3a], $45-59 \mathrm{ml} / \mathrm{min} / 1.73 \mathrm{~m}^{2}$; stage 3b [G3b], 30-44 ml/min $/ 1.73 \mathrm{~m}^{2}$; stage 4 [G4], 15-29 ml/ $\mathrm{min} / 1.73 \mathrm{~m}^{2}$; stage 5 [G5], <15 ml/min/1.73m²); and UACR levels from spot urine specimens (normal [A1]: $<30 \mathrm{mg} / \mathrm{g}$; microalbuminuria [A2]: $30-299 \mathrm{mg} / \mathrm{g}$; macroalbuminuria [A3]: $\geq 300 \mathrm{mg} / \mathrm{g}$ ).

Patients were categorized based on the test results available on the index date. If results on the index date were available for only 1 of the 2 tests (eGFR or UACR), the value closest to the index date was used for the other test. Patients with results for 1 test only were categorized based on that test and assigned to the missing category for the other test. Costs were also estimated separately for patients with a full year of follow-up and those who died during the first year.

All statistical analysis was performed using the Aetion Evidence Platform, version 3.7 (Aetion, New York, NY). ${ }^{23}$

\section{Results}

\section{PARTICIPANTS AND PATIENT CHARACTERISTICS}

Within the study period, 106,369 patients with T2D and newly recognized CKD were identified (Figure 2). The mean (SD) age was 70.6 (12.9) years; $56.5 \%$ of patients were female; and $57.7 \%$ of patients were White. Approximately $73 \%$ of the cohort were Medicare Advantage patients.

The mean (SD) and median [interquartile range] number of days of follow-up among the commercial health plan patients were 300 (109) and 365 [255-365] days, respectively, and among the Medicare Advantage patients was

\section{TABLE 1 Baseline Demographic Characteristics and Comorbidities of T2D Patients with Newly Recognized CKD Identified in Optum CDM, January 1, 2008-December 31, 2017}

\begin{tabular}{|c|c|c|}
\hline & \multicolumn{2}{|c|}{$\begin{array}{c}\text { T2D Patients with } \\
\text { Newly Recognized } \\
\text { CKD Diagnosis } \\
N=106,369\end{array}$} \\
\hline \multicolumn{3}{|l|}{ Demographics on cohort entry date } \\
\hline \multicolumn{3}{|l|}{ Age (years) } \\
\hline Mean (SD) & 70.6 & $(12.9)$ \\
\hline Median [IQR] & \multicolumn{2}{|c|}{$71.0 \quad[63.0-78.0]$} \\
\hline Gender male, n (\%) & 46,263 & $(43.5)$ \\
\hline \multicolumn{3}{|l|}{ Race, n (\%) } \\
\hline White & 61,398 & $(57.7)$ \\
\hline Black & 11,865 & $(11.2)$ \\
\hline Asian & 3,769 & $(3.5)$ \\
\hline Hispanic & 13,912 & $(13.1)$ \\
\hline Unknown or missing & 15,425 & $(14.5)$ \\
\hline \multicolumn{3}{|l|}{ Region, n (\%) } \\
\hline Northeast & 10,535 & (9.9) \\
\hline Midwest & 10,261 & (9.6) \\
\hline South & 60,978 & $(57.3)$ \\
\hline West & 24,191 & $(22.7)$ \\
\hline Missing or unknown & 404 & $(0.4)$ \\
\hline \multicolumn{3}{|l|}{ Laboratory values on cohort entry date } \\
\hline eGFR value $\left(\mathrm{ml} / \mathrm{min} / 1.73 \mathrm{~m}^{2}\right)$, median [IQR] & \multicolumn{2}{|c|}{$53.0 \quad[47.0-58.0]$} \\
\hline UACR value $(\mathrm{mg} / \mathrm{g})$, median [IQR] & \multicolumn{2}{|c|}{$63.4[35.5-148.3]$} \\
\hline \multicolumn{3}{|l|}{ Index CKD stage ${ }^{b}$} \\
\hline \multicolumn{3}{|l|}{ eGFR stage, n (\%) } \\
\hline Stage 1 & 5,696 & $(5.4)$ \\
\hline Stage 2 & 8,026 & $(7.6)$ \\
\hline Stage $3 a$ & 63,243 & $(59.5)$ \\
\hline Stage 3b & 15,400 & $(14.5)$ \\
\hline Stage 4 & 1,758 & $(1.7)$ \\
\hline Stage 5 & 201 & $(0.2)$ \\
\hline Missing & 12,045 & $(11.3)$ \\
\hline \multicolumn{3}{|l|}{ UACR category, n (\%) } \\
\hline Al & 12,310 & $(11.6)$ \\
\hline $\mathrm{A} 2$ & 27,926 & $(26.3)$ \\
\hline A3 & 3,714 & $(3.5)$ \\
\hline Missing & 60,439 & $(56.8)$ \\
\hline Deyo comorbidity score, mean (SD) & 2.28 & $(1.52)$ \\
\hline
\end{tabular}




\section{TABLE 1}

\begin{tabular}{|c|c|c|}
\hline & \multicolumn{2}{|c|}{$\begin{array}{c}\text { T2D Patients with } \\
\text { Newly Recognized } \\
\text { CKD Diagnosis } \\
\text { N=106,369 }\end{array}$} \\
\hline \multicolumn{3}{|l|}{ Comorbidities during baseline, $n$ (\%) } \\
\hline Angina pectoris & 25,709 & $(24.2)$ \\
\hline Acute coronary syndrome & 2,913 & $(2.7)$ \\
\hline Anemia & 19,761 & $(18.6)$ \\
\hline Atrial fibrillation & 10,873 & $(10.2)$ \\
\hline Chronic lung/pulmonary disease & 20,818 & $(19.6)$ \\
\hline Coronary artery disease & 14,363 & $(13.5)$ \\
\hline Heart failure & 11,795 & $(11.1)$ \\
\hline Hyperkalemia & 2,257 & $(2.1)$ \\
\hline Hypokalemia & 3,528 & $(3.3)$ \\
\hline Hyponatremia & 2,305 & $(2.2)$ \\
\hline Hyperlipidemia & 88,552 & $(83.2)$ \\
\hline Hypertension & 95,108 & $(89.4)$ \\
\hline Microvascular disease & 34,176 & $(32.1)$ \\
\hline Myocardial infarction & 1,862 & $(1.8)$ \\
\hline Pain disorders & 73,459 & $(69.1)$ \\
\hline Peripheral vascular disease & 16,711 & $(15.7)$ \\
\hline Resistant hypertension ${ }^{c}$ & 16,889 & $(15.9)$ \\
\hline Sleep apnea & 11,588 & $(10.9)$ \\
\hline Stroke & 4,603 & (4.3) \\
\hline
\end{tabular}

aPatients with ESRD, CKD, kidney transplant, dialysis, or conditions other than diabetes that may cause CKD during the 365-day baseline period were excluded.

${ }^{b}$ Stage 1 , eGFR $\geq 90 \mathrm{ml} / \mathrm{min} / 1.73 \mathrm{~m}^{2}$ and UACR $\geq 30 \mathrm{mg} / \mathrm{g}$; stage 2 , eGFR $60-89 \mathrm{ml} / \mathrm{min} / 1.73 \mathrm{~m}^{2}$ and UACR $\geq 30 \mathrm{mg} / \mathrm{g}$; stage $3 a$, eGFR $45-59 \mathrm{ml} / \mathrm{min} /$ $1.73 \mathrm{~m}^{2}$; stage $3 b$, eGFR $30-44 \mathrm{ml} / \mathrm{min} / 1.73 \mathrm{~m}^{2}$; stage 4, eGFR $15-29 \mathrm{ml} /$ $\mathrm{min} / 1.73 \mathrm{~m}^{2}$; stage $5,<15 \mathrm{ml} / \mathrm{min} / 1.73 \mathrm{~m}^{2} ; \mathrm{Al}$, UACR $<30 \mathrm{mg} / \mathrm{g}$ and $e \mathrm{GFR}$ $<60 \mathrm{ml} / \mathrm{min} / 1.73 \mathrm{~m}^{2} ; \mathrm{A} 2$, UACR $30-299 \mathrm{mg} / \mathrm{g} ; \mathrm{A} 3$, UACR $\geq 300 \mathrm{mg} / \mathrm{g}$. 'Resistant hypertension is defined as 4 or more unique antihypertensive medication prescription claims within a 30-day period.

$C K D=$ chronic kidney disease; eGFR = estimated glomerular filtration rate; $E S R D=$ end-stage renal disease; $I Q R=$ interquartile range; $T 2 D=$ type 2 diabetes mellitus; $U A C R=$ urinary albumin-creatinine ratio.

317 (793) and 365 [324-365] days, respectively. Among commercial health plan patients, 35.1\% had fewer than 365 days of follow-up, and among Medicare Advantage patients, $28.8 \%$ had fewer than 365 days of follow-up.

The most common comorbidities were hypertension (89.4\%), followed by hyperlipidemia (83.2\%) and pain disorders (69.1\%). Common cardiovascular-related comorbidities included angina pectoris (24.2\%), RH (15.9\%), coronary artery disease (13.5\%), HF (11.1\%), and atrial fibrillation (10.2\%). The majority of patients were identified at CKD stage 3 (74\%; Table 1). The number of patients within each KDIGO eGFR/UACR strata can be found in Supplementary Materials 2: Tables and Figures, Table 4 (available in online article).

\section{HEALTH CARE RESOURCE UTILIZATION}

T2D patients with newly recognized CKD had on average 0.3 inpatient, $0.7 \mathrm{ER}$, and 12.6 outpatient visits PPPY. The mean duration of all inpatient stays per patient during follow-up was 3.2 days (Table 2 ).

T2D patients with newly recognized CKD and comorbid HF had an almost 3 times higher mean count of inpatient visits PPPY, a 3 times longer average duration of inpatient stay, and double the count of ER visits compared with patients without HF. T2D patients with newly recognized CKD and comorbid anemia had double the mean number of hospitalizations PPPY and 2 times longer duration of inpatient stay compared with those without anemia (Table 2).

The mean count of inpatient, outpatient, and ER visits PPPY was higher among T2D patients with newly recognized CKD at more advanced CKD stages, as captured by the KDIGO classification. The mean count of inpatient visits PPPY ranged from approximately 0.2 to 1.2 across 5 eGFR and 3 UACR categories, showing rates of hospitalizations up to 7-fold higher among those in advanced stages of the disease compared with those in early disease stages. Additionally, the mean total duration of all inpatient visits was 10 times longer among those with CKD stage 5 compared with CKD stage 1 . The mean number of outpatient and ER visits PPPY gradually increased almost 3-fold across eGFR and UACR categories (results for HCRU by KDIGO classification are found in Tables 10-14 in Supplementary Materials 2: Tables and Figures, available in online article).

\section{COST}

For the overall population of T2D patients with newly recognized CKD, the mean annualized total cost was $\$ 24,029$ PPPY. Mean costs by type were $\$ 7,223$ PPPY for inpatient, $\$ 5,087$ PPPY for outpatient, \$1,073 PPPY for ER visits, and $\$ 4,672$ PPPY for pharmacy cost.

T2D patients with newly recognized CKD and HF or anemia incurred total annualized costs of \$41,951 and $\$ 33,127$ PPPY, respectively. The mean annualized costs among patients with HF were higher than the costs among patients without $\mathrm{HF}$ in all categories: 2.6 times for inpatient, 


\section{TABLE 2 Annualized HCRU and Cost of T2D Patients with Newly Recognized CKD Identified in Optum CDM, January 1, 2008-December 31, 2017}

\begin{tabular}{l|c|c|c|c}
\hline \multicolumn{1}{|c|}{$\begin{array}{c}\text { T2D } \\
\text { Patients } \\
\text { with CKD } \\
\mathbf{n = 1 0 6 , 3 6 9}\end{array}$} & $\begin{array}{c}\text { T2D } \\
\text { Patients } \\
\text { with CKD } \\
\text { and HF } \\
\mathbf{n = 1 1 , 7 9 5}\end{array}$ & $\begin{array}{c}\text { T2D } \\
\text { Patients } \\
\text { with CKD } \\
\text { and RH } \\
\mathbf{n = 1 6 , 8 8 9}\end{array}$ & $\begin{array}{c}\text { T2D } \\
\text { Patients } \\
\text { with CKD } \\
\text { and Anemia } \\
\mathbf{n = 1 9 , 7 6 1}\end{array}$ \\
\hline HCRU outcomes, mean count PPPY & 0.3 & 0.7 & 0.4 & 0.5 \\
\hline Inpatient visits & 12.6 & 18.4 & 14.1 & 16.2 \\
\hline Outpatient visits & 0.7 & 1.3 & 0.8 & 0.9 \\
\hline Emergency room visits & 3.2 & 7.4 & 4.0 & 5.3 \\
\hline Total duration of inpatient stays (days) & \multicolumn{3}{l}{} \\
\hline Cost measures, mean cost PPPY (USD) & 24,029 & 41,952 & 28,266 & 33,127 \\
\hline Total cost & 7,222 & 16,116 & 9,009 & 11,338 \\
\hline Inpatient cost & 5,087 & 8,281 & 5,834 & 6,693 \\
\hline Outpatient care cost ${ }^{b}$ & 1,073 & 2,108 & 1,249 & 1,490 \\
\hline Emergency room costs & 4,672 & 5,706 & 5,815 & 5,438 \\
\hline Pharmacy costs & 5,975 & 9,741 & 6,359 & 8,168 \\
\hline Other ${ }^{c}$
\end{tabular}

apatients with ESRD, CKD, kidney transplant, or dialysis during the 365-day baseline period were excluded. ${ }^{b}$ Costs incurring from office visits for primary care and specialty and dialysis centers, home infusion, longterm care, and skilled nursing settings are captured in the analysis as outpatient cost.

"Other costs include claims with missing values for "type of service," as well as those that did not fit the individual categories, including urgent care, durable medical equipment, drugs administered, home visits, services and supplies, transportation services, rehab/skilled facility, allergy testing, diagnostic testing, immunizations and injections, laboratory, mental health, obstetrics, pathology, professional other, radiology, surgery, vision/hearing/speech, and professional other.

$C D M=$ Clinformatics Data Mart; $C K D=$ chronic kidney disease; $E S R D=e n d$-stage renal disease;

$H C R U=$ health care resource utilization; $H F=$ heart failure; $P P P Y=$ per person per year; $R H=$ resistant hypertension; T2D = type 2 diabetes mellitus.

1.8 times for outpatient, and 2.2 times for ER. Among patients with anemia, the mean annualized inpatient cost was almost double that of those without anemia (\$11,338 PPPY and \$6,292 PPPY, respectively; Table 2; also see Table 15 in Supplementary Materials 2: Tables and Figures, available in online article).

Annualized total cost was consistently higher across worsening stages of CKD (Figure 3; also see Table 16 in Supplementary Materials 2: Tables and Figures, available in online article). The higher costs were driven mainly by inpatient costs. Within each stage of CKD defined by eGFR, the mean annualized total cost increased across groups of UACR (Figure 3). The most striking contrast across UACR categories was observed among patients with CKD stage 5 , where the total annualized cost was \$17,432 PPPY among those with normal UACR levels of $<30 \mathrm{mg} / \mathrm{g}$ and $\$ 110,210$ PPPY among patients with microalbuminuria (30$300 \mathrm{mg} / \mathrm{g})$. Similarly, among patients with microalbuminuria, the annualized total cost was $\$ 18,529$ PPPY in eGFR-based CKD stage 1 and $\$ 110,210$ PPPY in eGFR-based CKD stage 5.

A large difference in mean annualized outpatient costs was observed between CKD stage 4 and CKD stage 5, with the observed cost being up to 5-fold greater among patients in group CKD stage 5. Differences in outpatient costs were smaller across other groups. Annualized pharmacy cost did not vary notably by CKD severity. To explore this further, pharmacy cost estimates were stratified by payer type (commercial vs. Medicare Advantage). An escalation of annualized pharmacy cost was observed by CKD stage among patients on commercial insurance but not among those on Medicare. Among those with commercial insurance, the annualized pharmacy cost was $\$ 5,361$ among patients with CKD stage 1 and increased to $>\$ 14,400$ among those with CKD stage 5. Among those on Medicare, the cost fluctuated between $\$ 5,000$ and $\$ 6,000$.

While death is not fully captured in the Optum claims, approximately $2 \%$ of patients in our study population had a record of death in follow-up. The average follow-up time for these patients was 189 days, and the average cost during that time was approximately 4 times higher compared with patients who had a full year of follow-up. The largest difference in cost was attributed to the cost of hospitalization.

Additional figures and tables of results are available in Supplementary Materials 2: Tables and Figures (available in online article).

\section{Discussion}

CKD is one of the most common complications of T2D, with multimorbidity resulting in considerable health and economic burden. Administrative claims data can provide important insights into the burden of disease within a population and provide real-world evidence on management of health conditions. In this U.S.-based cohort study of T2D patients with newly recognized CKD (identified using diabetes 


\section{FIGURE 3 Annualized Total Cost PPPY Among T2D Patients with Newly Recognized CKD Identified in Optum CDM, January 1, 2008-December 31, 2017, Stratified by KDIGO CKD Stages ${ }^{a}$}

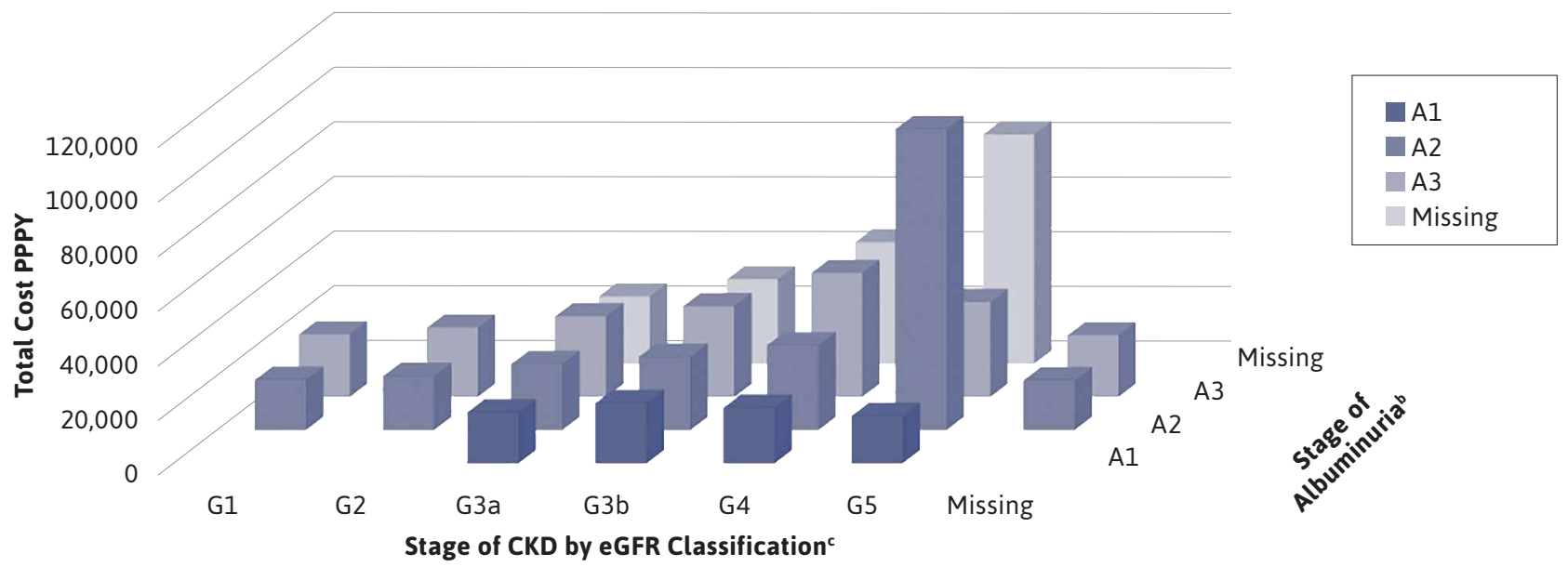

aPatients with ESRD, CKD, kidney transplant, or dialysis during the 365-day baseline period were excluded. See Table 16 in Supplementary Materials 2: Tables and Figures for full reporting (available in online article).

${ }^{b}$ Albuminuria stages: normal (A1), $<30 \mathrm{mg} / \mathrm{g}$; microalbuminuria (A2), 30-300 mg/g; macroalbuminuria (A3), >300 mg/g.

'CKD stages by eGFR classification: CKD stage 1 (G1), > $90 \mathrm{ml} / \mathrm{min} / 1.73 \mathrm{~m}^{2} ;$ CKD stage 2 (G2), 60-89 ml/min $/ 1.73 \mathrm{~m}^{2} ;$ CKD stage $3 a(G 3 a), 45-59 \mathrm{ml} / \mathrm{min} / 1.73 \mathrm{~m}^{2}$; CKD stage $3 b$ (G3b), 30-44 ml/min $/ 1.73 \mathrm{~m}^{2}$; CKD stage 4 (G4), $15-29 \mathrm{ml} / \mathrm{min} / 1.73 \mathrm{~m}^{2}$; CKD stage 5 (G5), $<15 \mathrm{ml} / \mathrm{min} / 1.73 \mathrm{~m}^{2}$.

$C D M=$ Clinformatics Data Mart; $C K D=$ chronic kidney disease; $E S R D=$ end-stage renal disease; $H C R U=$ health care resource utilization; $H F=$ heart failure; KDIGO = Kidney Disease: Improving Global Outcomes; $P P P Y=$ per person per year; $R H=$ resistant hypertension; $T 2 D=$ type 2 diabetes mellitus.

diagnosis claims and eGFR and UACR laboratory test results from administrative claims data), high HCRU and cost were observed, particularly among patients with more advanced CKD stages and among those with comorbid HF and anemia.

HCRU and cost were much higher among the subgroup of patients with HF or anemia, in which the mean annual number of inpatient and outpatient visits was almost double, and the mean duration of inpatient stay was almost 3 times longer. Total cost in these subgroups was almost 3 times higher PPPY compared with patients without the respective comorbidities. These findings are in line with those reported by Einarson et al. (2018) in a review study of international literature, estimating that patients with T2D and HF had a 59\% higher cost compared with those without. ${ }^{24}$ Polson et al. (2017) found that patients with CKD and HF had nearly triple the total health care costs compared with CKD patients without HF. ${ }^{15}$

Additionally, gradually increasing HCRU and cost were observed across worsening severity of CKD as indicated by KDIGO categories. A 7 times higher mean annual number of inpatient and outpatient visits and a 5 times greater annualized cost were observed among those with advanced CKD stages compared with those in early stages of CKD. Previous studies have also reported that HCRU and cost increase across severity of albuminuria and CKD stage. Zhou et al. (2017) reported that the annual number and duration of inpatient stays were 3 times higher, and total annual cost was more than 2 times higher, among diabetes patients with macroalbuminuria compared with those with normal albumin levels; however, eGFR levels were not evaluated. ${ }^{25}$ McBrien et al. (2013) reported that among Canadian patients with diabetes, adjusted cost was 5-fold higher for patients with eGFR $<15 \mathrm{~mL} / \mathrm{min} / \mathrm{m}^{2}$ compared with those with eGFR $>90 \mathrm{~mL} / \mathrm{min} / \mathrm{m}^{2}$ (\$115,348 vs. \$25,316) and was twice as high in patients with heavy proteinuria compared with those without (\$46,836 vs. \$24,531). ${ }^{26}$

Other studies also found that even early stages of kidney disease are associated with substantial medical cost, with costs increasing as disease severity worsens. ${ }^{27}$ The disproportionately high cost among patients with CKD stage 5 may be explained by high rates of dialysis, which has been estimated to cost $\$ 89,000$ per patient annually in the United States. ${ }^{28}$ Of patients captured at stage 4 and stage 5 in baseline ( $n=1,959$ or $1.9 \%$ of the population), $20 \%$ of patients had evidence of kidney transplant and $68 \%$ of dialysis events in follow-up.

Annualized pharmacy cost did not vary notably by CKD severity, although costs stratified by commercial and Medicare 
Advantage payer types differed. Pharmacy costs were relatively stable among patients with Medicare Advantage across CKD stages, whereas large differences in cost were observed across CKD stages among those with commercial insurance. These differences may be due to subsidized treatment for kidney disease among Medicare beneficiaries. ${ }^{29}$

Patients with comorbid T2D and CKD face challenges with timely diagnosis and disease management. Despite the elevated risk of CKD among diabetic patients, CKD screening with eGFR and UACR is suboptimal, resulting in the underdiagnoses of CKD. While clinicians appear to recognize advance stages of CKD compared with mild CKD stages, such delays in diagnosis and treatments result in higher rates of disease progression and poor clinical outcomes. ${ }^{30,31}$ The KDIGO treatment guidelines recommend use of an angiotensin-converting enzyme inhibitor or an angiotensin II receptor blocker in patients with T2D and CKD to delay disease progression. ${ }^{32}$

Pharmacological treatment with antihyperglycemic medication in high-risk diabetic patients is also complex. Several cardiovascular safety trials have revealed preventative renal effects among antidiabetic medications, including sodium-glucose cotransporter-2 inhibitors and glucagonlike peptide-1 receptor agonists. ${ }^{33-37}$ However, treatment with antihyperglycemic medication among patients with coexistent CKD and HF or patients with advance CKD poses clinical challenges, with continual dose adjustments, step-wise additions, and treatment switching, as well as contraindication concerns. ${ }^{38}$ In addition, 2 large, international, randomized placebo-controlled trials are underway to evaluate the use of a mineralocorticoid receptor antagonist in lowering kidney failure, disease progression, and cardiovascular morbidity and mortality among patients with diabetic kidney disease. ${ }^{39,40}$

For advanced kidney disease, the KDIGO clinical guidelines recommend kidney transplants and dialysis for patients with severe kidney function (i.e., eGFR $<20 \mathrm{ml} /$ min/1.73 $\mathrm{m}^{2}$ and $5-10 \mathrm{ml} / \mathrm{min} / 1.73 \mathrm{~m}^{2}$, respectively). ${ }^{41}$ Our study showed increasing HCRU and cost among patients across worsening CKD severity and patients with multimorbidity, underscoring the benefits of early CKD diagnosis and adequate treatment to slow disease progression of CKD, prevent clinical outcomes, and reduce high health care expenditures.

Our study has several strengths. We identified T2D patients from U.S. administrative claims using a clinically meaningful algorithm, and CKD was further identified using laboratory test values, which are considered the gold standard when confirming this condition.

We demonstrated that the clinical criteria for CKD can be successfully applied to a large administrative claims dataset to identify patients of interest and reduce the potential for patient misclassification. Unlike evidence generated by clinical trials with strict eligibility criteria and treatment regimens, claims data reflect care that patients receive in a real-world setting, as well as how patients receive treatments and interact with the health care system. Additionally, the large population size allows for findings that are more generalizable to the broader group of patients.

Finally, the availability of both eGFR and UACR test results allowed for the stratification of estimates according to the KDIGO classification, which to our knowledge has not been previously done in a large real-world database.

In general, many HCRU and cost analyses require 365 days of follow-up as a study inclusion criteria; however, this method may lead to selection bias, since patients who disenroll or die during that year of follow-up are excluded from the analyses. Such an approach will largely underestimate costs among patients with high morbidity and mortality, such as patients with more advanced T2D who develop CKD and suffer from other comorbidities. Our study did not require a minimum follow-up time, but instead reported annualized HCRU and costs PPPY.

Given the strengths of this analysis, the findings for HCRU and cost among this population of T2D patients with newly recognized CKD further support the conclusion that earlier diagnosis of CKD and prevention of comorbidities and disease progression among this population may help to create significant reductions in HCRU and costs.

\section{LIMITATIONS}

This study had several limitations common to analyses using administrative claims databases. First, laboratory results were available for approximately $30 \%$ of patients included in the overall Optum CDM database, reducing the sample size due to use of a more specific eligibility criteria for newly recognized CKD that required at least 2 eGFR or UACR laboratory results..$^{42}$ However, the use of laboratory results for patient selection increased the likelihood of identifying true CKD patients, given the suboptimal performance of ICD-9/10-CM codes. In addition, this data source lacked indirect cost of care and did not capture the overall burden on patients and caregivers, such as effect on quality of life; however, claims data provide valuable evidence about direct medical costs of managing their health conditions.

Second, claims data carry a potential for misclassification of patients' diagnoses, since the presence of a diagnosis code on a claim may not indicate the presence of a disease, but a rule-out code. To address this limitation in identifying patients with T2D, a validated algorithm was used. ${ }^{43}$ 
Third, while eGFR and UACR laboratory results are referred to as the gold standard for identification of kidney disease, since they reflect established definitions used in clinical care, ${ }^{4,44,45}$ there are circumstances in which any creatinine-based estimate of kidney function, including eGFR, may be less reliable. The measurement of urine albumin and eGFR is also known to be imprecise..$^{20,44}$ To address this limitation, at least 2 laboratory results at least 3 months apart in the CKD range were required.

Fourth, by reporting annualized HCRU and cost outcomes calculated at the population level, the degree of variation of estimates was not assessed (e.g., SDs, medians, or interquartile ranges). Despite this limitation, the approach used offered several advantages, such as allowing patients with less than 1 year of follow-up (including those who died) to remain in the analysis, thus, reducing selection bias that would have resulted from the inclusion of healthier patients.

Finally, while this database is representative of the commercially insured U.S. population, and comprises a large proportion of Medicare Advantage patients (73\%), it may not be representative of non-U.S.-based patients and noncommercially insured populations in the United States.

\section{Conclusions}

While CKD typically develops many years after the initial diagnosis of diabetes, it may also be present at the time of T2D diagnosis. ${ }^{31,46}$ Despite the high burden of CKD among patients with T2D, many patients are unaware of their condition and face delays in CKD diagnosis and proper treatments. ${ }^{47}$ This study indicates that CKD patients with more advanced disease and other comorbidities have much higher HCRU and cost. Given these findings, it is possible that earlier diagnosis of
CKD and its complications in patients with T2D, as well as interventions that are effective in halting or slowing the progression of CKD and treating other comorbid conditions, could result in substantial cost savings. The net cost benefit of such interventions will need to be assessed in future studies.

\section{DISCLOSURES}

This study was funded by Bayer. Kelly is an employee of, and owns stock options in, Aetion, which was contracted by Bayer to conduct the study. Petruski-Ivleva was an employee of Aetion during the planning, analysis, and interpretation stages of the study. Kovesdy received honoraria from Amgen, Astra Zeneca, Bayer, Cara Therapeutics, Reata, Takeda, and Tricida. Fried received consultant fees from Bayer, Novo Nordisk, and Bristol-Meyers Squibb. Folkerts, Blankenburg, and Gay are Bayer employees.

This work was presented as a poster at the annual European Association for the Study of Diabetes (EASD) conference held in Barcelona, Spain, on September 16-20, 2019.

\section{ACKNOWLEDGMENTS}

The authors thank Pattra Mattox, SM, CMPP, for her medical writing support for this work.

\section{REFERENCES}

1. Centers for Disease Control and Prevention. National diabetes statistics report, 2017. Accessed November 5, 2020. https://dev.diabetes.org/sites/default/files/ 2019-06/cdc-statistics-report-2017.pdf

2. Piette J D, Kerr E A. The impact of comorbid chronic conditions on diabetes care 2006. Diabetes Care. 2006;29(3);72531. doi:10.2337/diacare.29.03.06.dc05-2078 3. American Diabetes Association. Economic costs of diabetes in the U.S. in 2017. Diabetes Care. 2018; 41(5):917-28.

4. Saran R, Li Y, Robinson B, et al. United States Renal Data System 2014 annual data report: epidemiology of kidney disease in the United States. Am J Kidney Dis. 2015;66(1 Suppl 1):Svii, S1-305. doi: 10.1053/j.ajkd.2015.05.001
5. Fernandez B, Elewa U, Sanchez-Nino MD, et al. 2012 update on diabetic kidney disease: the expanding spectrum, novel pathogenic insights and recent clinical trials. Minerva Med. 2012;103(4):219-34.

6. Tuttle K, Bakris GL, Bilous RW, et al. Diabetic kidney disease: a report from an ADA Consensus Conference. Diabetes Care. 2014;37(10):2864-83. doi: 10.2337/ dc14-1296

7. Afkarian M, Zelnick LR, Hall YN, et al. Clinical manifestations of kidney disease among U.S. adults with diabetes, 19882014. JAMA. 2016;316(6):602-10.

8. de Boer IH, Rue TC, Hall YN, et al. Temporal trends in the prevalence of diabetic kidney disease in the United States. JAMA. 2011;305(24):2532-39.

9. United States Renal Data System. Chapter 6: Healthcare expenditures for persons with CKD. In: 2017 USRDS Annual Data Report. Volume 1 - CKD in the United States. 2017. National Institutes of Health, National Institute of Diabetes and Digestive and Kidney Diseases, Bethesda, MD. Accessed November 5, 2020. https:// www.usrds.org/media/1675/v1 c06 medexp 17.pdf

10. Kovesdy CP. Epidemiology of hyperkalemia: an update. Kidney Int Suppl (2011). 2016;6(1):3-6.

11. National Institute of Diabetes and Digestive and Kidney Diseases. Anemia in chronic kidney disease. July 1, 2014. Accessed November 5, 2020. https:// www.niddk.nih.gov/health-information/ kidney-disease/anemia

12. Foley RN, Parfrey PS, Sarnak MJ. Clinical epidemiology of cardiovascular disease in chronic renal disease. Am J Kidney Dis. 1998;32(5):S112-S119.

13. Gilbert RE, Connelly K, Kelly DJ, et al. Heart failure and nephropathy: catastrophic and interrelated complications of diabetes. Clin J Am Soc Nephrol. 2006;1(2):193-208.

14. Steigerwalt, S. Management of hypertension in diabetic patients with chronic kidney disease. Diabetes Spectr. 2008;21(1):30-36.

15. Polson, Michael, Todd C. Lord, Kangethe A, et al. Clinical and economic impact of hyperkalemia in patients with chronic kidney disease and heart failure. J Manag Care Spec Pharm. 2017;23(Suppl 4-a):S2-S9. doi: 10.18553/jmcp.2017.23.4-a.s2a 
16. Optum. Optum Clinformatics Data Mart. 2017. Accessed November 16, 2020. https://www.optum.com/content/ dam/optum/resources/productSheets/ Clinformatics for Data Mart.pdf

17. Kern EFO, Maney M, Miller DR, et al. Failure of ICD-9-CM codes to identify patients with comorbid chronic kidney disease in diabetes. Health Serv Res. 2006;41(2):564-80. doi: 10.1111/j.14756773.2005.00482.x

18. American Diabetes Association. 11 Microvascular complications and foot care: standards of medical care in diabetes-2019. Diabetes Care. 2019;42(Suppl 1):S124-S138. doi: 10.2337/dc19-S011.

19. Vassalotti JA, Centor R, Turner BJ, et al. practical approach to detection and management of chronic kidney disease for the primary care clinician. Am J Med. 2016;129(2):153-62.e7. doi: 10.1016/j. amjmed.2015.08.025

20. KDIGO CKD Work Group. 2012 Clinical Practice Guideline for the Evaluation and Management of Chronic Kidney Disease. Kidney Int Suppl. 2013;3(1):1-150. Accessed November 5, 2020. https://kdigo.org/wpcontent/uploads/2017/02/KDIGO_2012_ CKD GL.pdf

21. Ruzafa J, Paczkowski R, Boye KS, et al. Estimated glomerular filtration rate progression in UK primary care patients with type 2 diabetes and diabetic kidney disease: a retrospective cohort study. Int J Clin Pract. 2015;69(8):871-82.

22. United States Renal Data System. Volume 1: CKD analytical methods. In: 2018 USRDS Annual Data Report. Volume 1: CKD in the United States. Accessed November 16, 2020. https://www. usrds.org/media/1726/v1 c10 ckdmethods_18_usrds.pdf

23. Wang SV, Verpillat P, Rassen JA, et al. Transparency and reproducibility of observational cohort studies using large healthcare databases. Clin Pharmacol Ther 2016;99(3):325-32. doi:10.1002/cpt.329

24. Einarson TR, Acs A, Ludwig C, et al. Economic burden of cardiovascular disease in type 2 diabetes: a systematic review. Value Health. 2018;21(7):881-90.

25. Zhou Z, Chaudhari P, Yang H, et al. Healthcare resource use, costs, and disease progression associated with diabetic nephropathy in adults with type 2 diabetes: a retrospective observational study. Diabetes Ther. 2017;8(3):555-71.
26. McBrien KA, Manns BJ, Chui B, et al. Health care costs in people with diabetes and their association with glycemic control and kidney function. Diabetes Care. 2013;36(5):1172-80.

27. Honeycutt AA, Segel JE, Zhuo X, et al. Medical costs of CKD in the Medicare population. J Am Soc Nephrol. 2013;24(9):1478-83.

28. Collins AJ, Foley RN, Chavers B, et al. Volume 2: Atlas of End-Stage Renal Disease in the United States. In: USRDS 2013 Annual Data Report. Am J Kidney Dis. 2014;63(1 Suppl):e151-e478. doi: 10.1053/ S0272-6386(13)01541-2

29. Howell BL, Powers CA, Weinhandl ED, et al. Sources of drug coverage among Medicare beneficiaries with ESRD. J Am Soc Nephrol. 2012;23(5):959-65. doi: 10.1681/ASN.2011070740

30. Szczech LA, Stewart RC, Su HL, et al. Primary care detection of chronic kidney disease in adults with type-2 diabetes: The ADD-CKD Study (awareness, detection and drug therapy in type 2 diabetes and chronic kidney disease). PLoS One. 2014;9(11):e110535

31. American Diabetes Association. Standards of medical care in diabetes-2018. Diabetes Care. 2018;41(Suppl 1): S1-S159.

32. National Kidney Foundation KDOQI Clinical Practice Guideline for Diabetes and CKD: 2012 Update. Am J Kidney Dis. 2012;60(5):850-86. doi: 10.1053/ j.ajkd.2012.07.005.

33. Wanner C, Lachin JM, Inzucchi SE, et al. Empagliflozin and clinical outcomes in patients with type 2 diabetes, established cardiovascular disease and chronic kidney disease. Circulation. 2018;137(2):119-29.

34. Neal B, Perkovic V, Matthews DR, et al. Canagliflozin and cardiovascular and renal events in type 2 diabetes. $\mathrm{N}$ Engl J Med. 2017;377(21):644-57.

35. Mann JFE, Ørsted DD, BrownFrandsen K, et al. Liraglutide and renal outcomes in type 2 diabetes. $\mathrm{N}$ Engl J Med. 2017;377(9):839-84

36. Marso SP, Bain SC, Consoli A, et al. Semaglutide and cardiovascular outcomes in patients with type 2 diabetes. N Engl J Med. 2016;375(19):1834-44.

37. Mahaffey KW, Jardine MJ, Bompoint S, et al. Canagliflozin and cardiovascular and renal outcomes in type 2 diabetes mellitus and chronic kidney disease in primary and secondary cardiovascular prevention groups. Circulation. 2019;140(9):739-50.
38. American Diabetes Association. 9. Pharmacologic approaches to glycemic treatment: standards of medical care in diabetes 2020. Diabetes Care. 2020;43(Suppl. 1):S98-S110. doi: 10.2337/ dc20-S009.

39. Bakris GL, Agarwal R, Anker SD, et al. Design and baseline characteristics of the Finerenone in Reducing Kidney Failure and Disease Progression in Diabetic Kidney Disease Trial. J Nephrol. 2019;50(5):333-44. doi: 10.1159/000503713

40. Ruilope LM, Agarwal R, Anker SD, et al. Design and Baseline Characteristics of the Finerenone in Reducing Cardiovascular Mortality and Morbidity in Diabetic Kidney Disease Trial. Am J Nephrol. 2019;50(5):345-56. doi: 10.1159/000503712

41. Inker LA, Astor BC, Fox CH, et al. KDOQI U.S. commentary on the 2012 KDIGO clinical practice guideline for the evaluation and management of CKD. Am J Kidney Dis. 2014;63(5):713-35.

42. Optum. Clinformatics Data Mart. User manual, version 7.0. 2017.

43. Miller DR, Safford MM, Pogach LM. Who has diabetes? Best estimates of diabetes prevalence in the Department of Veteran Affairs based on computerized patient data. Diabetes Care. 2004;27 (Suppl 2):b10-b21.

44. Levey AS, Inker LA, Matsushita K, et al. GFR decline as an end point for clinical trials in CKD: a scientific workshop sponsored by the National Kidney Foundation and the U.S. Food and Drug Administration. Am J Kidney Dis. 2014;64(6):821-35. doi:10.1053/j. ajkd.2014.07.030

45. Menke A, Casagrande S, Geiss L, et al. Prevalence of and trends in diabetes among adults in the United States, 19882012. JAMA. 2015;314(10):1021-29. doi: 10.1001/jama.2015.10029

46. Gatwood J, Chisholm-Burns M, Davis R, et al. Evidence of chronic kidney disease in veterans with incident diabetes mellitus. PLoS One. 2018;13(2):e0192712. doi:10.1371/journal.pone.0192712

47. Agrawal V, Jaar BG, Frisby XY, et al. Access to health care among adults evaluated for CKD: findings from the Kidney Early Evaluation Program (KEEP). Am J Kidney Dis. 2012; 59(3 Suppl 2):S5-S15. 